\title{
Investigation on MDOF Bilateral Teleoperation Control System Using Geared DC-Motor
}

\author{
Lee Jun Wei ${ }^{1}$, Ahmad Zaki bin Hj Shukor ${ }^{1} \&$ Muhammad Herman bin Jamaluddin ${ }^{1}$ \\ ${ }^{1}$ Center for Robotic and Industrial Automation (CeRIA), Faculty of Electrical Engineering, Universiti Teknikal \\ Malaysia Melaka, Durian Tunggal, Malaysia \\ Correspondence: Lee Jun Wei, Faculty of Electrical Engineering, Universiti Teknikal Malaysia Melaka, Durian \\ Tunggal, Malaysia. Tel: 6013-632-8128. E-mail: junwei1690@gmail.com
}

\author{
Received: May 16, 2016 \\ Accepted: June 20, 2016 \\ Online Published: July 29, 2016 \\ doi:10.5539/mas.v10n11p54 \\ URL: http://dx.doi.org/10.5539/mas.v10n11p54
}

\begin{abstract}
This paper presents the research on bilateral teleoperation control system of two link planar manipulator. The bilateral control system consists of master and slave system using geared DC-motor. Both master and slave manipulators are actuated by DC-Micromotor attached to planetary gearhead to increase the output torque. In the previous researches, the common used actuators were linear motors and direct drive DC motor. However, the application of DC motor with gearhead is vast in industry, due to the need for high output force or torque. Thus in this paper, a deeper research on bilateral teleoperation control system is proposed with implementation of gear into the DC-motor. The modelling of multi-degrees-of-freedom (MDOF) bilateral teleoperation control system is designed with the implementation of workspace observer (WOB), reaction force observer (RFOB), position controller and force controller. During the experiments, free motion and contact motion were conducted to validate the proposed setup in bilateral teleoperation control system. The position and torque responses of both master and slave manipulators were observed. The operationality and reproducibility of this proposed system were evaluated through experimental results. Ultimately, this paper focused on the performance of the proposed setup and is analysed by using reproducibility and operationality.
\end{abstract}

Keywords: haptics, MDOF bilateral teleoperation control system, geared DC-motor, operationality, reproducibility

\section{Introduction}

Haptic is one of the developing technology in the robotic application. Bilateral teleoperation with master and slave system is one of the haptic technology. This is to enable human to feel haptic information from the remote environment which human cannot present to. This technique is expected to apply in many situations such as space, surgery and in industrial environment. For example, development of bilateral teleoperation control system in surgery for surgical tools and instruments has been researched for the past few decades. As a result, this technology assisted in minimal invasive surgeries for mankind. Thus, K. Prasanga et al. and K. Tanida et al. proposed and developed haptic surgical forceps that can be used as surgery tools (Prasanga et al., 2012; Tanida et al., 2012). With the bilateral control system in this device, the surgeon can feel the stiffness of the tissues and react accordingly.

If the bilateral teleoperation control system has multi-degrees-of-freedom, disturbance observer can be designed in two kinds of domain, which is joint space and workspace. Joint space is the implementation of actuator at each joint and workspace is the implementation of end effector of the manipulator (Murakami et al., 1995). The disturbance observer implemented in workspace control is called as workspace observer. H. Tai proved that using workspace based bilateral control is better than joint space in terms of stability and transparency of equivalent mass matrix based bilateral control of MDOF (Tai \& Murakami, 2010; Lasnier \& Murakami, 2010). In terms of stability, the joint space which included inverse Jacobian matrix deteriorated when approaching singularity point. Indeed, the workspace control scheme is the most convenient for human because it describes the desired position and motion imposed by the human. Additionally, with the implementation of this scheme and workspace observer into MDOF bilateral control, the external forces exerted on master and slave system can be physically fealt. More analysis on workspace-based bilateral control with MDOF motion system and the studies on reproducibility and operationality of the MDOF bilateral control system were also researched (Horie 


\section{\& Murakami, 2011).}

The acceleration-based bilateral control method was able to generate improvement in performance even in MDOF bilateral control system. A robust control based on disturbance observer approach improve the motion control system with the equivalent mass matrix determined arbitrarily and independent of configuration and inertia variation of the manipulator (Murakami et al., 1995). However, the posture change in the manipulator can cause fluctuation of cut-off frequency of the workspace observer due to the variation of the equivalent mass in workspace (Shimono \& Yamashita, 2012). Moreover, further investigation in paper (Togashi et al., 2014) stated that the control performance can be improve by implementing the use of the equivalent mass matrix including the non-diagonal elements.

In particular, geared motor has been used vastly in industrial environment such as factory automation, robotics, industrial machines and medical sciences as well as laboratory technology. Geared motor also brings cutting-edge technology applications such as outer-space and underwater exploration, advanced robotics, and machinery. The advantage of geared motor is the ability to produce high torque. To produce high torque from a motor itself without gear for power transmission, the size of the motor has to be large. This in fact is not just costly, but also impractical in size. Thus, it is important to realise that the higher the torque required, the larger the size of the motor. With much different type of gears and ratio, the engineer can decide the torque output for an application.

However, in the past approaches, researches on bilateral teleoperation control system utilised linear motor. The output force is powerful but the cost is high. Moreover, it controls linear (Cartesian) positions. Nevertheless, some researches approached bilateral teleoperation control system using DC rotational motor. But the output power is very low. For instance, the implementation of geared motor in bilateral teleoperation control system is a new step in research. The outcome of the teleoperation using geared motor was able to realize a low cost teleoperation system. Unfortunately, geared motor produces large joint friction in teleoperation and affects the force/torque sensorless control. As a result, this also affects the reproducibility and operationality of a bilateral control system.

Furthermore, for industrial robots seen in automation and car manufacturing, the operation of the robots is programed based on trajectory position. The robots are usually are for pick and place or assembly task. Yet, operation area is fenced around to avoid human from accessing the operation zone. This is because the industrial robots are rigid and sensor-less to external environment, thus it will harm and injure humans when it contacts with human during operation. Consequently, these industrial robots are not safe during operation and not human friendly. In order to be safer, accessible and human friendly, the system must have external force feedback from the environment other than operation task. By all means if the system is able to track external force, the industrial robots will halt the operation immediately when it makes contact with the undesired force from the environment. Thus, this research focused on two link rotational planar manipulator for bilateral teleoperation control system. Moreover, this research also highlights the application of gearhead on the DC-motor not just to increase the output torque, but also to achieve low cost teleoperation system.

This paper is organized as follows. The first part introduces about the bilateral teleoperation control system followed by the workspace observer (WOB) and reaction force observer (RFOB) for two DOF planar bilateral teleoperation manipulator. Next the experimental setup and experimental manipulations is demonstrated. At the result and discussion section, the experiments of free and contact motions conducted were discussed. Ultimately, the performance of the proposed setup is analysed by using reproducibility and operationality.

\section{Bilateral Teleoperation Control System}

The dynamic modelling of workspace control for two DOF planar manipulator has been introduced in (Wei et al., 2015). In this paper, the combination of of workspace control and bilateral control system were presented. In this system, there are two MDOF manipulators. Both manipulators have the same two DOF, which are distinguished as master and slave manipulator system. Both systems are interconnected as bilateral control system. Figure 2.1 shows the block diagram of two link planar manipulator bilateral control based on acceleration control. The actuator information from joint space is transformed into human modal space and the bilateral control is implemented at each joint. Then the acceleration reference is transformed to actuator information in joint space. 


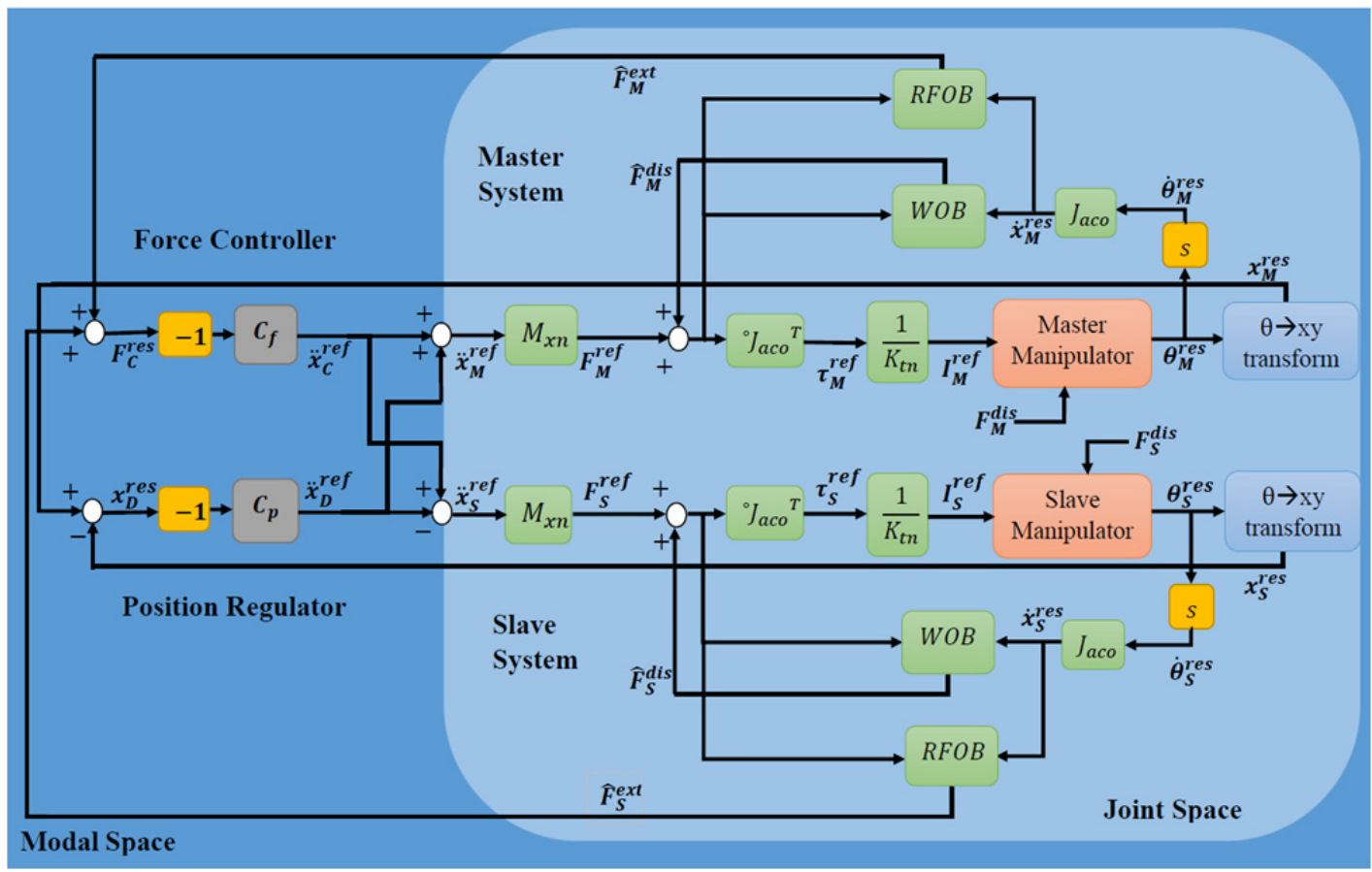

Figure 2.1 Block diagram of two link planar manipulator bilateral control based on acceleration control

In order to transfer information in haptic communication, the realization of position tracking and the law of action and reaction between master system and slave system are important. The $F_{M}$ represents the force applied by human operator to the master manipulator while the $F_{S}$ represents the force applied by the environment at the slave manipulator. Moreover, vector force is applied at the end effector (in $x$ and $y$ coordinate) of both master and slave manipulator. Thus the force on master and slave consists of $F_{M x}, F_{M y}$ and $F_{S x}, F_{S y}$, respectively. Equation (2.1) represents the summation of the action force from human operator $F_{M}$ and reaction force from environment $F_{S}$ which should converge to zero. On the contrary, Equation (2.2) represents that the position error in Cartesian space between master position $x_{M}, y_{M}$ and slave position $x_{S}, y_{S}$ should converge to zero. $\hat{F}_{M}^{e x t}$ and $\hat{F}_{S}^{\text {ext }}$ are estimated using RFOB rather than obtaining it from the force sensor. The estimated force from master and slave manipulators' end effector also in Cartesian space $\hat{F}_{M x}^{\text {ext }}, \hat{F}_{M y}^{\text {ext }}, \hat{F}_{S x}^{\text {ext }}$ and $\hat{F}_{S y}^{\text {ext }}$ Equations (2.2) to (2.5).

$$
\begin{gathered}
\mathrm{F}_{\mathrm{C}}^{\mathrm{res}}=\hat{\mathrm{F}}_{\mathrm{M}}^{\text {ext }}+\hat{\mathrm{F}}_{\mathrm{S}}^{\text {ext }} \\
\mathrm{x}_{\mathrm{D}}^{\text {res }}=\mathrm{x}_{\mathrm{M}}^{\text {res }}-\mathrm{x}_{\mathrm{S}}^{\text {res }} \\
\mathrm{F}_{\mathrm{C}}^{\mathrm{res}}=0 \\
\mathrm{x}_{\mathrm{D}}^{\text {res }} \rightarrow 0 \\
\mathrm{x}_{\mathrm{D}}^{\text {res }}=0
\end{gathered}
$$

The basic concept of bilateral motion control system on both master and slave system is required to be implemented in its total position in differential mode $x_{D}$ and total force in common mode $F_{C}$, on both $x$ and $y$ coordinate (Ohnishi et al., 2010). The common mode and the differential mode are able to be designed independently. In order to decouple between these two modes, the second order Hadamard matrix (quarry matrix), $H_{2}$ is applied as modal decomposition as shown in Equation (2.6) (" * " denotes non-zero element and not taken into account for bilateral control system). Thus, position control and force control are achieved simultaneously.

$$
\begin{gathered}
{\left[\begin{array}{cc}
\mathrm{F}_{\mathrm{C}}^{\mathrm{res}} & * \\
* & \mathrm{x}_{\mathrm{D}}^{\mathrm{res}}
\end{array}\right]=\left[\begin{array}{cc}
1 & 1 \\
1 & -1
\end{array}\right]\left[\begin{array}{ll}
\hat{\mathrm{F}}_{\mathrm{M}}^{\text {ext }} & \mathrm{x}_{\mathrm{M}}^{\mathrm{res}} \\
\hat{\mathrm{F}}_{\mathrm{S}}^{\text {ext }} & \mathrm{x}_{\mathrm{S}}^{\text {res }}
\end{array}\right]} \\
=\mathrm{H}_{2}\left[\begin{array}{ll}
\hat{\mathrm{F}}_{\mathrm{M}}^{\text {ext }} & \mathrm{x}_{\mathrm{M}}^{\mathrm{res}} \\
\hat{\mathrm{F}}_{\mathrm{S}}^{\text {ext }} & \mathrm{x}_{\mathrm{S}}^{\text {res }}
\end{array}\right]
\end{gathered}
$$

Then the system includes the force controller $C_{f}$ and position controller $C_{p}$ to calculate the common mode 
acceleration reference, $\ddot{x}_{C}^{r e f}$ and differential mode acceleration reference, $\ddot{x}_{D}^{\text {ref }}$ using the transformed force and position response as shown in Equation (2.7) and Equation (2.8), respectively;

$$
\begin{aligned}
& \ddot{\mathrm{x}}_{\mathrm{C}}^{\text {ref }}=-\mathrm{C}_{\mathrm{f}} \mathrm{F}_{\mathrm{C}}^{\text {res }} \\
& \ddot{\mathrm{x}}_{\mathrm{D}}^{\text {ref }}=-\mathrm{C}_{\mathrm{p}} \mathrm{X}_{\mathrm{D}}^{\text {res }}
\end{aligned}
$$

The position and force information are transformed into acceleration reference so that both information can be controlled together. By using the Equation (2.7), (2.8) and second-order inverse Hadamard matrix, $H_{2}^{-1}$, the reference signal in acceleration dimension to control master and slave are shown Equation (2.9).

$$
\begin{aligned}
{\left[\begin{array}{c}
\ddot{\mathrm{X}}_{\mathrm{M}}^{\mathrm{ref}} \\
\ddot{\mathrm{X}}_{\mathrm{S}}^{\mathrm{ref}}
\end{array}\right] } & =\frac{1}{2}\left[\begin{array}{cc}
1 & 1 \\
1 & -1
\end{array}\right]\left[\begin{array}{l}
\ddot{\mathrm{X}}_{\mathrm{C}}^{\mathrm{ref}} \\
\ddot{\mathrm{X}}_{\mathrm{D}}^{\mathrm{ref}}
\end{array}\right] \\
& =\mathrm{H}_{2}^{-1}\left[\begin{array}{l}
\ddot{\mathrm{X}}_{\mathrm{C}}^{\mathrm{ref}} \\
\ddot{\mathrm{X}}_{\mathrm{D}}^{\mathrm{ref}}
\end{array}\right]
\end{aligned}
$$

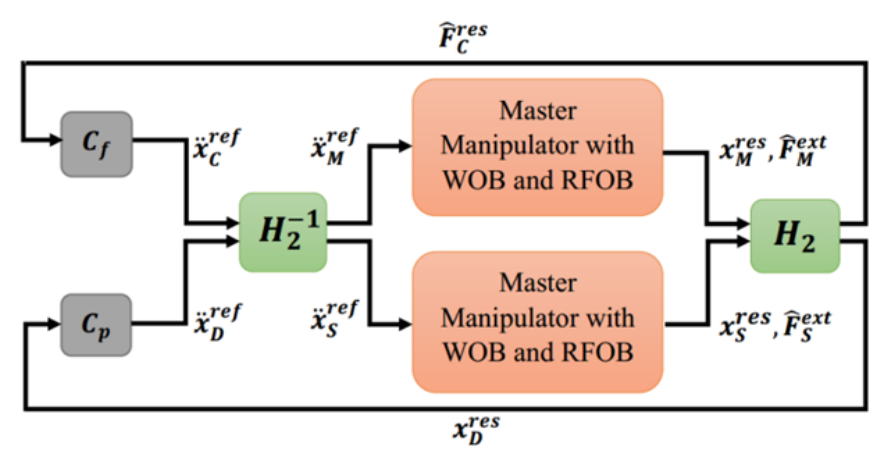

Figure 2.2 General acceleration based four channel bilateral controller

Figure 2.2 shows the block diagram of four-channel bilateral controller of this system. From the above system, high transparency is achieved between master system and slave system of bilateral control system. Human operator at master side can feel an environment in the slave side as if it is in the master side.

The function of the position controller system is to allow the tracking position system to track the desired trajectory in the critically damp response as shown in Equation (2.12). The position coefficient and velocity coefficient are set based on the natural angular frequency and a damping coefficient of the control system as show in Equation (2.10) and Equation (2.11) (Jamaluddin et al., 2014). Moreover, the force controller system has to maintain the contact stability between force at end-effectors and the force at the contact object (Leksono et al., 1996) as shown in Equation (2.13).

$$
\begin{gathered}
\mathrm{K}_{\mathrm{p}}=\omega_{\mathrm{n}}^{2} \\
\mathrm{~K}_{\mathrm{d}}=2 \delta \omega_{\mathrm{n}} \\
\mathrm{C}_{\mathrm{p}}=\mathrm{K}_{\mathrm{p}}+\mathrm{K}_{\mathrm{d}} \mathrm{S} \\
\mathrm{C}_{\mathrm{f}}=\mathrm{K}_{\mathrm{f}}
\end{gathered}
$$

\subsection{Disturbance Observer (DOB) and Reaction Force Observer (RFOB)}

In the previous paper has mentioned on the implementation of workspace observer into Direct Cartesian scheme and showed improvement in the workspace control (Wei et al., 2015). Although bilateral control system is used in this paper, the meaning and implementation of WOB are the same. However, reaction force observer (RFOB) is used too. Figure 2.3 shows the block diagram of the implementation of WOB and RFOB into the MDOF bilateral control system. Equation (2.17) and Equation (2.18) are the estimated disturbance $\hat{F}^{\text {dis }}$ for master and slave system and both $x$ axis and $y$ axis, respectively. 


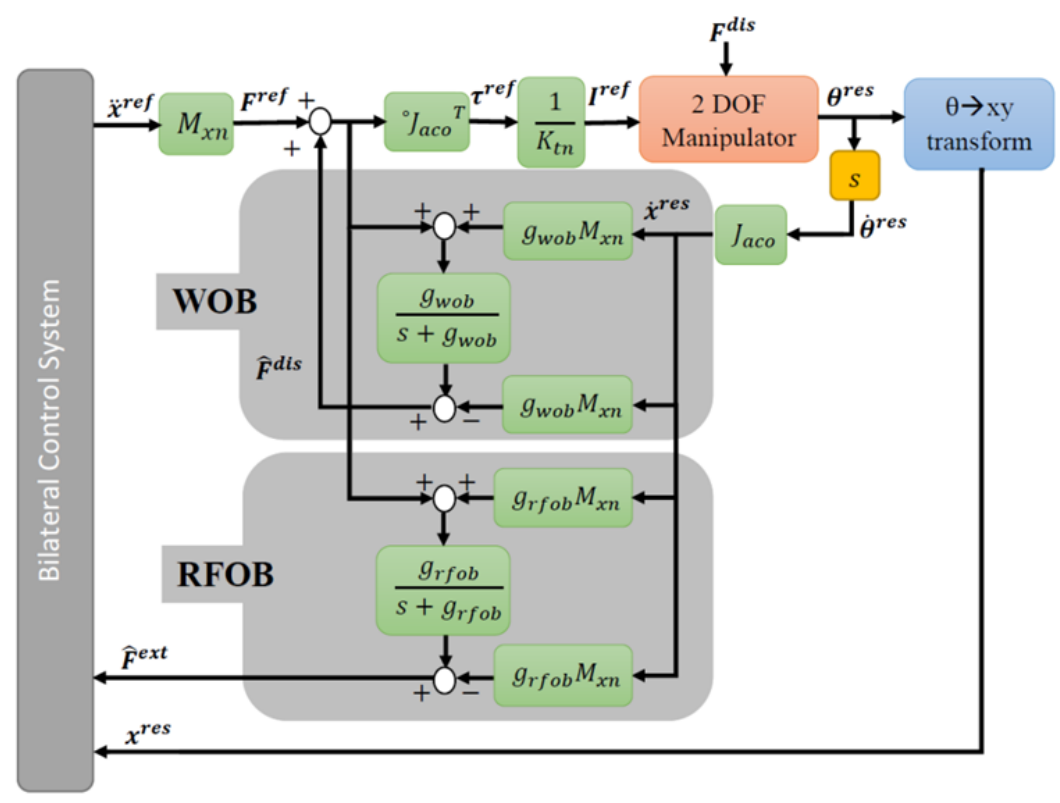

Figure 2.3 Block diagram of workspace based disturbance observer and reaction force observer

$$
\begin{aligned}
& \mathrm{F}=\mathrm{F}^{\mathrm{ref}}-\mathrm{F}^{\mathrm{dis}} \\
& \mathrm{F}^{\mathrm{dis}}=\mathrm{F}^{\mathrm{int}}+\mathrm{F}^{\mathrm{ext}}+\mathrm{F}^{c}+\mathrm{D} \dot{\theta}^{\text {res }} \\
& \hat{F}^{\text {dis }}=\frac{g_{w o b}}{s+g_{w o b}}\left(F^{r e f}+\hat{F}^{d i s}+g_{w o b} M_{x n} \dot{x}\right)-g_{w o b} M_{x n} \dot{x}
\end{aligned}
$$

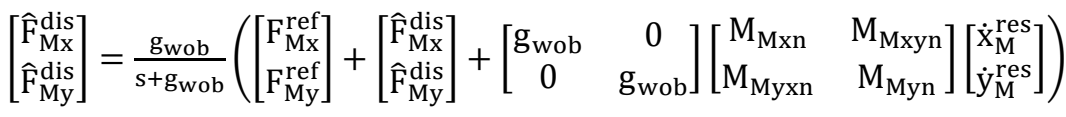

$$
\begin{aligned}
& -\left[\begin{array}{cc}
g_{w o b} & 0 \\
0 & g_{w o b}
\end{array}\right]\left[\begin{array}{ll}
M_{M x n} & M_{M x y n} \\
M_{M y x n} & M_{M y n}
\end{array}\right]\left[\begin{array}{l}
\dot{x}_{M}^{\text {res }} \\
\dot{y}_{M}^{\text {res }}
\end{array}\right]
\end{aligned}
$$

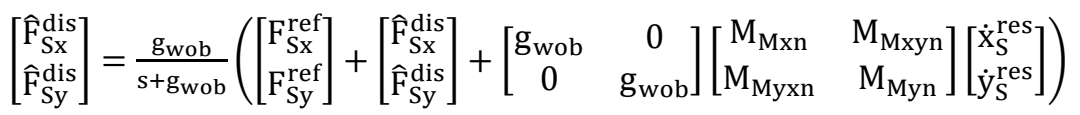

$$
\begin{aligned}
& -\left[\begin{array}{cc}
g_{w o b} & 0 \\
0 & g_{w o b}
\end{array}\right]\left[\begin{array}{ll}
M_{M x n} & M_{M x y n} \\
M_{M y x n} & M_{M y n}
\end{array}\right]\left[\begin{array}{l}
\dot{X}_{S}^{\text {res }} \\
\dot{y}_{S}^{\text {res }}
\end{array}\right]
\end{aligned}
$$

$F^{\text {dis }}$ is the total disturbance force generated in workspace for both $x$ axis and $y$ axis where;

$F^{\text {int }}$ Internal force;

$F^{c}$ Coulomb friction;

$D \dot{\theta}^{\text {res }} \quad$ Viscous force;

$$
\begin{gathered}
\hat{\mathrm{F}}^{\text {dis }}=\frac{\mathrm{g}_{\mathrm{wob}}}{\mathrm{s}+\mathrm{g}_{\mathrm{wob}}} \mathrm{F}^{\text {dis }} \\
\frac{\mathrm{g}_{\mathrm{wob}}}{\mathrm{s}+\mathrm{g}_{\mathrm{wob}}}
\end{gathered}
$$

The relationship between real disturbance force and estimated disturbance force is as described in Equation (2.19). By using the workspace observer, the system was able to estimate the disturbance force through a low-pass filter as shown in Equation (2.20), where $g_{w o b}$ is a cut-off frequency. The bandwidth of the WOB low-pass filter is set as high as possible to estimate a wide frequency range of disturbance. However, it is limited by practical and robustness constraints (Ishii et al., 2007).

Furthermore, by subtracting the external disturbances and system uncertainties from input of a WOB, it can estimate the reaction force applied to the system. It is necessary to identify them as precisely as possible. This process is called as reaction force observer (RFOB) (Murakami \& Ohnishi, 1993) as shown is Figure 2.3. Equation (2.21) shows that the reaction force observer is estimated through first-order LPF. RFOB can estimate external 
force without force sensor because force sensor has many drawbacks. The study of comparison between force sensor and reaction force observer based on force control system has been analysed (Sarlyildiz \& Ohnishi, 2014).

$$
\widehat{\mathrm{F}}^{\mathrm{ext}}=\frac{\mathrm{g}_{\mathrm{rfob}}}{\mathrm{s}+\mathrm{g}_{\mathrm{rfob}}} \mathrm{F}^{\mathrm{ext}}
$$

Where $\frac{g_{r f o b}}{s+g_{r f o b}}$ is the low-pass filter (LPF) and $g_{r f o b}$ is a cut-off frequency.

However, identification of frictional force is needed in advance to estimate the external torque. The friction disturbance can be compensated by DOB only if an accurate friction model is provided. Here, the frictional force is not compensated and cannot be ignored. Thus later in the experiments, the system has friction effects largely from planetary gear. The friction force largely contributed as an operational force. However, the compensation of friction force is not in the scope.

\subsection{Hardware Setup}

There are two sets of two link planar manipulators. Each joint is actuated by a planetary geared DC-Micromotor with incremental encoder. It has 5000ppr (pulse per revolution) before gearhead. The links are designed with a length of $0.13 \mathrm{~m}$ each with a base attached to a platform to prevent any unwanted vibration. The link can be either used to operate the system by human operator on master side or to the environmental contact on the slave side. The links at both master and slave side are in horizontal orientation that provide zero gravitational effects. Thus, only disturbance effect and frictional force are present in the gearhead and motor. Moreover, the main purpose of this setup is to investigate the operationality torque and reproducibility of this proposed geared bilateral teleoperation control system.

Planetary gearhead is able to provide higher torque for a low torque DC-Mircomotor. Moreover, the backlash is crucial to the haptic application where it can affect the performance of the bilateral system. The output of master and slave manipulator is position measured by the encoders mounted at the back of each motor shaft. The velocity response is obtained by derivative of position response and noise from the signal is filtered away by Low Pass Filter (LPF) in the control loop in the computer software. Within the Simulink, the processed data is set to analogue voltage reference signal from the Micro-Box to the motor driver. Motors are driven by Maxon motor driver (ESCON 50/5) in current-based control mode. The reference value represents the desired current that the motor driver will inject to the motor. The motor torque is directly proportional to the motor current (Hace \& Jezernik, 2010). Figure 2.4 shows the experimental setup of this research.

Nevertheless, before conducting the experiment, the position controller gains, force controller gain, bandwidth of DOB and RFOB/RTOB are stochastically tune in order to achieve the required performance of the particular system. These gains are tuned based on random trials until the overall system is logically stable and shows good performance (Wei et al., 2016).

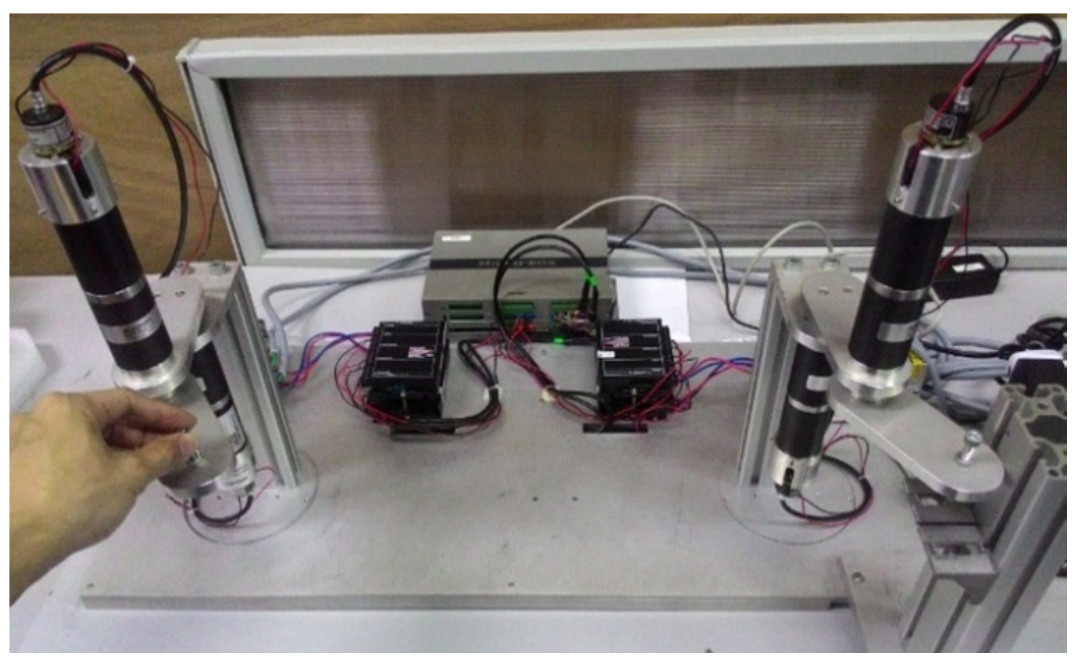

Figure 2.4 Experimental Setup 


\subsection{Experimental Manipulations}

In this part, there are two experiments to be conducted. The first experiment is free motion and the second experiment is contact motion. During the free motion, the human operator control freely (without any obstacle) at master system while the slave system also follow freely without contact with any obstacle. During the contact motion, the initial position of the end-effector of the slave system is very near to the surface of the objects, but not touching it yet. The human operator operates the master manipulator manually in order for the slave manipulator to contact the object. During contact motion, human operator holds the master handle and moves the handle while the slave handle is constrained by a static object. The human operator then applies torque at the master handle. The hard object is an aluminium while the soft object is a sponge. Specifically, the human operator applied force on $y$ axis at the end-effector of master manipulator during contact motion as shown in Figure 2.5.

The force and position response from both master and slave system are recorded, for both free motion and contact motion experiments. The position responses from master and slave system are obtained from rotary encoders while the external force applied to the master and slave systems are estimated by RFOB. The force and position response from both master and slave system are compared with each other to validate the operationality and reproducibility of the bilateral teleoperation control system. Nevertheless, the operationality can be validated from the free motion experiment while the reproducibility can be validated from the contact motion experiment. Then, the evaluation of this part of experiment is explained in the results section.

Here, singularity is not cancelled. For this case, the singularity points according to end-effector are when the second joint (elbow) is at $0^{\circ}$ or $180^{\circ}$.

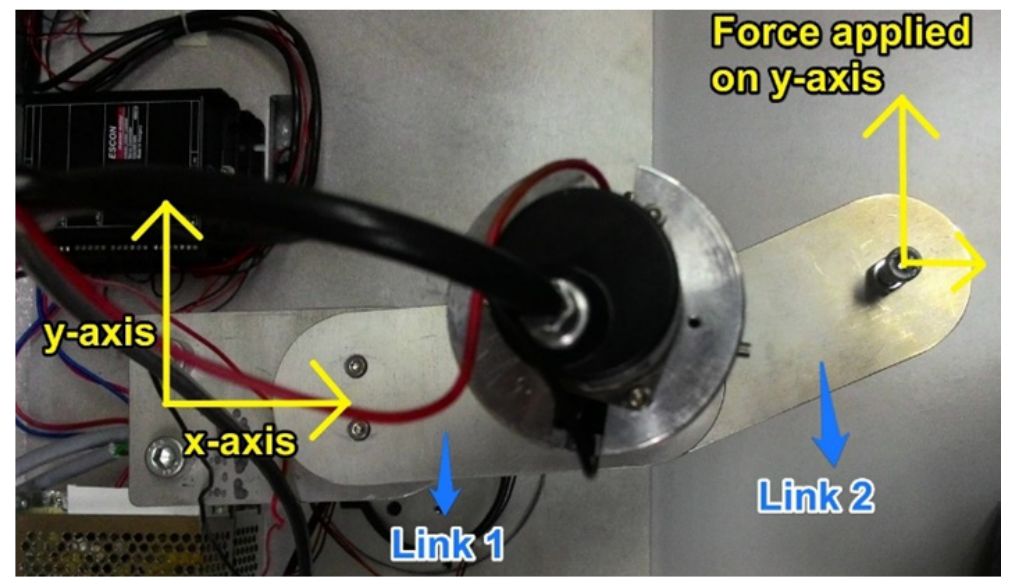

Figure 2.5 Direction of applied force at the end-effector of master manipulator (top view)

\section{Results}

Table 1. Parameters in Experiment

\begin{tabular}{lll}
\hline Parameter & \multicolumn{1}{c}{ Description } & \multicolumn{1}{c}{ Value } \\
\hline$l_{1}$ & Link 1 & $0.13 \mathrm{~m}$ \\
$l_{2}$ & Link 2 & $0.13 \mathrm{~m}$ \\
$M_{n 1}$ & Nominal mass 1 & $1.085 \mathrm{~kg}$ \\
$M_{n 2}$ & Nominal mass 2 & $0.19 \mathrm{~kg}$ \\
$K_{t n}$ & Nominal torque constant & $0.7164 \mathrm{Nm} / \mathrm{A}$ \\
$K_{p}$ & Position gain & 10000 \\
$K_{d}$ & Velocity gain & 200 \\
$K_{f}$ & Force gain & 2 \\
$N_{1}: N_{2}$ & Master gear ratio & $36: 1$ \\
$g_{d o b}$ & Cut-off frequency of disturbance observer & $50 \mathrm{rad} / \mathrm{s}$ \\
$g_{r t o b}$ & Cut-off frequency of reaction force observer & $50 \mathrm{rad} / \mathrm{s}$ \\
\hline
\end{tabular}


In this part of the experiment, the master and slave manipulator are identical two link planar manipulator. The length of the two link $\mathrm{l}_{1}$ and $\mathrm{l}_{2}$, geared DC-motor at each joint are identical for both master and slave manipulator. Nevertheless, the nominal mass $M_{n 1}$ and $M_{n 2}$ for master and slave manipulator are identical. The experiments are conducted with the parameters shown in Table 1.

\subsection{During Free Motion}

In this section, the free motion experiment is conducted. Free motion means that the human operator manipulates the master manipulator freely without any obstacle and the slave manipulator also does not contact anything. This experiment is to investigate the operational torque of this bilateral teleoperation control system during free motion. Operationality is degree of operational force which human operator feels besides reaction force from the environment which is desired for comfortable operation for human operator (Iida \& Ohnishi, 2004). Figure 3.1 shows free motion experiment result while Figure 3.2 shows the XY trajectory response during free motion (noted that the graph shows the origin of master and slave manipulator are at the same local frame).
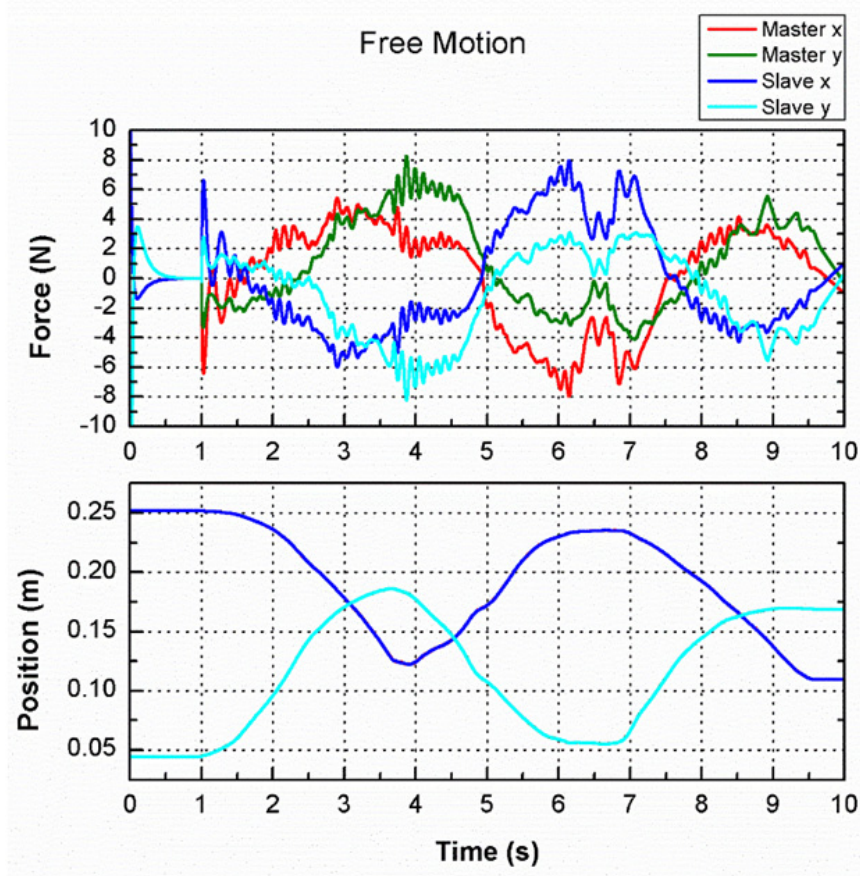

Figure 3.1 Force and position response during free motion

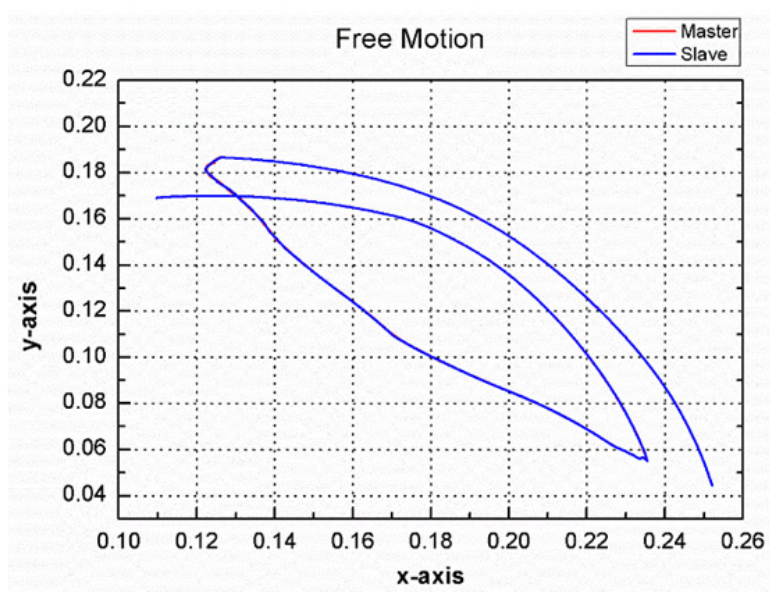

Figure 3.2 XY trajectory response during free motion

During the free motion, human operator need to apply some amount of force in order to overcome friction to 
move the master manipulator as shown in Figure 3.1. Human operator also needs to apply higher force when changing the direction of motion. The force to overcome friction (friction force) largely contributes as an operational force. However, the force for both $x$ and $y$ position tracked very well at both master and slave system. Thus, the law of action and reaction are achieved between master and slave system.

It is also shown the position response from both master and slave system are tracked perfectly as shown in Figure 3.1 where the red and blue line overlap each other for $x$ axis while green and cyan overlap each other for $y$ axis. Figure 3.2 shows the position response of XY trajectory for both master and slave manipulator during random free motion. Again, the position response from both master and slave system are tracked perfectly where the red and blue line overlap each other.

\subsection{During Contact Motion}

In this section, the contact motion experiment is conducted. The human operator makes three contact motions with the object within 10s. This experiment is to investigate the reproducibility of this bilateral teleoperation control system when it comes in contact with object. According to Figure 3.3 and Figure 3.5, contact motion on aluminium (hard object) and sponge (soft object) are operated manually by the human operator, respectively. Figure 3.4 and Figure 3.6 shows the XY trajectory response during contact motion on aluminium (hard object) and sponge (soft object) are also operated manually by human operator respectively (noted that the graph shows the origin of master and slave manipulator are at the same local frame).
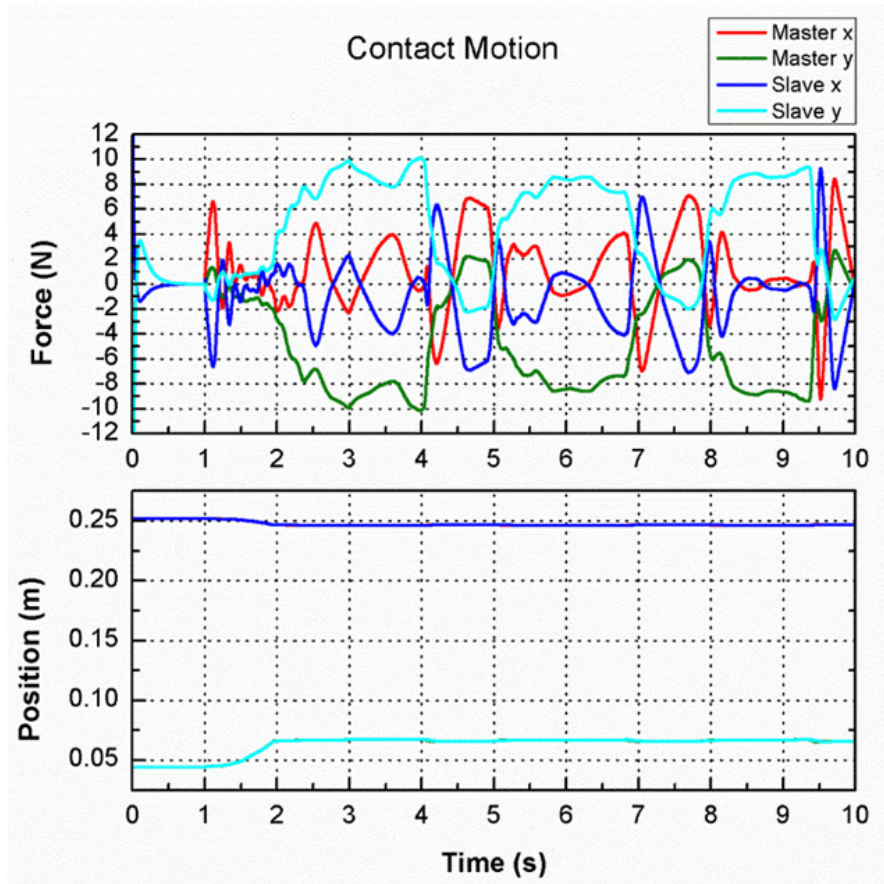

Figure 3.3 Force and position response during contact motion (hard object) 


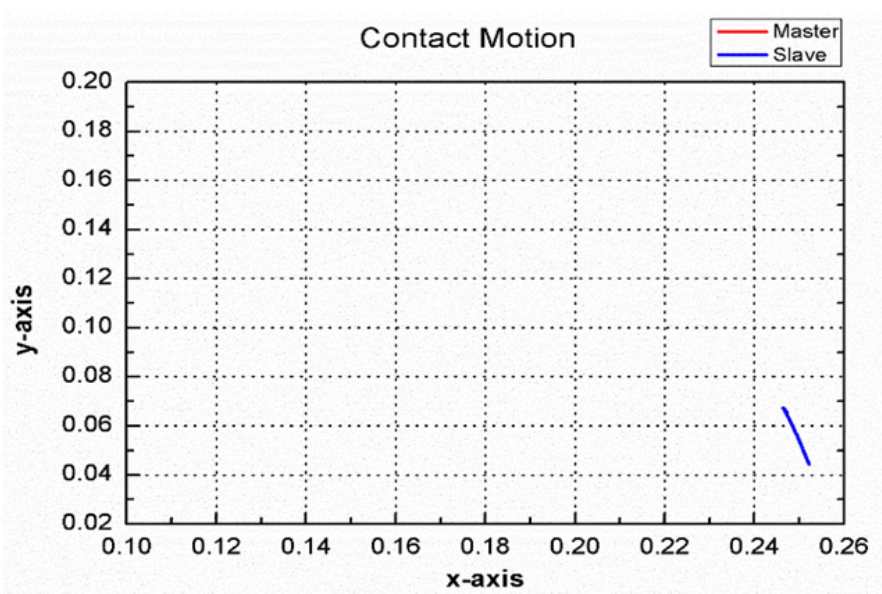

Figure 3.4 XY trajectory response during contact motion (hard object)
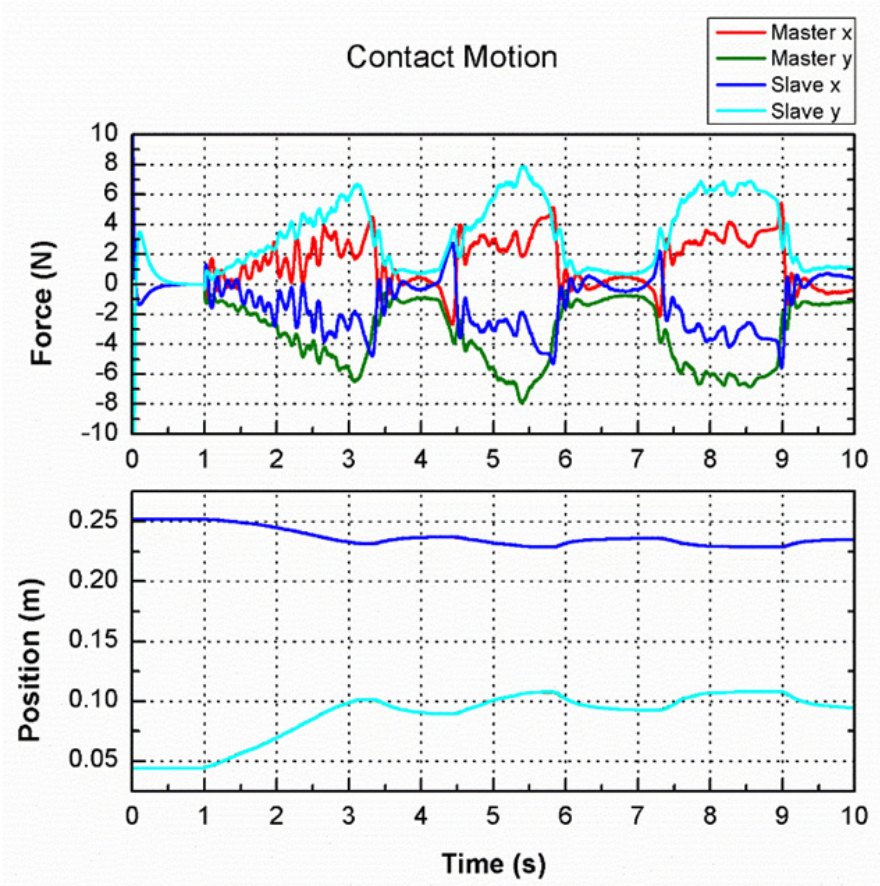

Figure 3.5 Force and position response during contact motion (soft object)

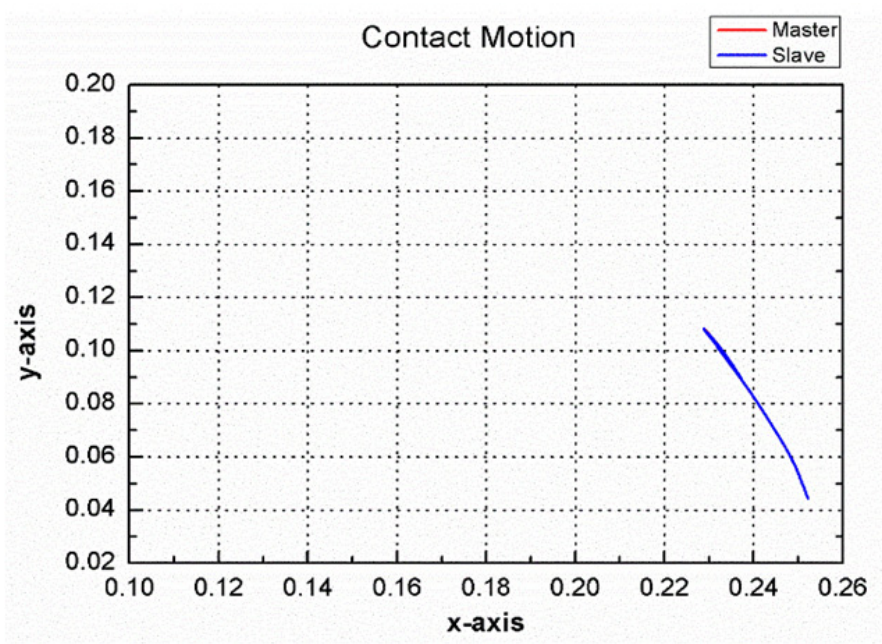

Figure 3.6 XY trajectory response during contact motion (soft object) 
During contact with aluminium, the force response between master and slave manipulator are tracked perfectly. The law of action and reaction force between master and slave system is realized in force response as shown in Figure 3.3.

It is shown that the position response from both master and slave system are tracked perfectly as shown in Figure 3.3 where the red and blue line overlap each other for $x$ axis while green and cyan overlap each other for $y$ axis. Figure 3.4 shows the position response of $\mathrm{XY}$ trajectory for both master and slave manipulator during random contact with the aluminium block. Again, the position response from both master and slave system are tracked perfectly where the red and blue line overlap each other.

Next, during contact with the sponge, the force and position response between master and slave manipulator are tracked perfectly. The law of action and reaction force between master and slave system is realized in force response as shown in Figure 3.5.

It can be seen that the position response from both master and slave system are tracked perfectly as shown in Figure 3.5 where the red and blue line overlap each other for $x$ axis while green and cyan overlap each other for $y$ axis. Figure 3.6 shows the position response of XY trajectory for both master and slave manipulator during random contact with the sponge. Again, the position response from both master and slave system are tracked perfectly where the red and blue lines overlap each other.

Meanwhile, when the human operator released force at the master manipulator, the sponge eventually expanded to regain its shape. During the expansion of the sponge, an expansion force from the sponge pushed the slave manipulator which is in contact with sponge. Pushing the master manipulator too causes changes in position response in both master and slave manipulator, especially in $y$ axis direction, as can be observed in Figure 3.5 and Figure 3.6. Then, the human operator was able to feel the expansion force applied by the sponge.

By observing the force response shown in Figure 3.3 and Figure 3.5, the force for $y$ axis at master and slave are higher compared to $x$ axis. This is because the human operator applied more force at $y$ axis during the contact motion as mentioned in the procedures. This also can be shown in position response where $y$ position deviated during the contact motion. Figure 3.4 and Figure 3.6 showed the trajectory motion where master and slave manipulator start from initial position and contact with the aluminium and sponge, respectively. In brief, human operator manipulated the master manipulator more in the $y$ axis direction.

\section{Discussion}

During the experiments on this bilateral teleoperation control system with free motion, the position tracking of both master and slave manipulators are tracked perfectly. However, there is a large operational force that human operator needed to perform in order to move the master and slave manipulator comfortably and easily. It is shown that the operational force is multiplied by gear ratio of the gearhead.

During contact motion, it is shown that both position and force tracking at both master and slave manipulators are well tracked. The reproducibility during contact motion is satisfied by this experiment. The stable contact of the slave manipulator is achieved on aluminium and sponge. The human operator can feel sharpness of reaction force from hard object. The friction force is almost neglected as there is no free motion during the contact motion of hard object.

However, during contact with the sponge, there is extra operational force to compress the sponge which is frictional force from gear. Then, during the expansion from the sponge, the expansion force needs to overcome the frictional force at the slave system to expand. Thus, human operator from master manipulator felt less expansion force from the sponge. Overall, the position tracking of the end-effector and the "law of action and reaction" are achieved between master and slave system in the workspace. This shows that the geared DC-motor performs well in contact motion and reproduce the reaction force from the environment and the bilateral teleoperation control system is achieved.

\section{Conclusion}

A two link planar bilateral teleoperation systems are developed where each joint is actuated by DC-Micromotor with planetary gearhead to increase the output torque at master and slave manipulator. WOB and RFOB are implemented in the system to compensate the disturbance and to estimate the external torque, respectively. The experiments are conducted to validate the bilateral teleoperation control system. The haptic information which is the position and torque information from both master and slave manipulators are plotted and compared. The operationality and reproducibility are analysed by conducting free and contact motion, respectively. Experimental results supported the theoretical background of the proposed setup and displayed accurate position and force tracking. As a result, the proposed two link planar bilateral teleoperation control system using geared 
DC-motor is promising for future bilateral teleoperation applications.

Furthermore, since the geared motor is used in every setup, the human operator felt the stiffness of the gear. The higher the gear reduction ratio, the stiffer the human operator feels. This leads to high operational force for a haptic device and is a disadvantage in this haptic system. Thus, compensation of frictional force within gear is important. Additionally, the singularity is a common issue in MDOF manipulator. It gives a limitation and precaution for a MDOF manipulator during operation, or even injury or damage to the human operator or the manipulator itself. Thus, methods to eliminate singularity are a priority in MDOF manipulator to fully utilise the structure without compromising the position and force performance of MDOF bilateral control teleoperation system.

As for the future work, this bilateral teleoperation control system could be extended to further study on the application on MDOF two link planar micro-macro bilateral teleoperation control system using geared DC-motor for scaling purposes.

\section{Acknowledgments}

Identify The Authors wish to express their thanks to the Universiti Teknikal Malaysia Melaka (UTeM) under the UTeM ZAMALAH scheme, UTeM Short Term Grant (PJP/2O13/FKE(5D)/S01171) and (PJP/2015/FKE(1D)/S01392).

\section{References}

Hace, A., \& Jezernik, K. (2010). Bilateral teleoperation by sliding mode control and reaction force observer. Proc. IEEE Int. Symp. Ind. Electron, 1809-1816. http://dx.doi.org/10.1109/ISIE.2010.5637717

Horie, R., \& Murakami, T. (2011). An analysis of a novel master-slave control and its application to MDOF motion systems. IECON Proceedings (Industrial Electronics Conference), 474-479. http://dx.doi.org/10.1109/IECON.2011.6119357

Iida, W., \& Ohnishi, K. (2004). Reproducibility and operationality in bilateral teleoperation. Proceedings of 8th IEEE International Workshop AMC, 217-222. http://dx.doi.org/10.1109/AMC.2004.1297669

Ishii, E., Nishi, H., \& Ohnishi, K. (2007). Improvement of performances in bilateral teleoperation by using FPGA. IEEE Transactions on Industrial Electronics, 54(4), 1876-1884. http://dx.doi.org/10.1109/TIE.2007.898295

Jamaluddin, M. H., Shimono, T., \& Motoi, N. (2014). An integration method between vision-based disturbance observer and bilateral haptic system for robust tracking of target object. IEEE 13th International Workshop on Advanced Motion Control (AMC), 2014(1), 723-728. http://dx.doi.org/10.1109/AMC.2014.6823370

Lasnier, A., \& Murakami, T. (2010). Workspace based force sensorless bilateral control with multi-degree-of-freedom motion systems. The 11th International Workshop on Advanced Motion Control, AMC, 583-588. http://dx.doi.org/10.1109/AMC.2010.5464065

Leksono, E., Murakami, T., \& Ohnishi, K. (1996). On hybrid position / force cooperative control of multimanipulator based on workspace disturbance observer. Proceedings of the IEEE 22nd Int. Conf. on Industrial Electronics, Control, and Instrumentation, 1873-1878. http://dx.doi.org/10.1109/IECON.1996.570758

Murakami, T. et al. (1995). A motion control strategy based on equivalent mass matrix in multidegree-of-freedom manipulator. IEEE Transactions on Industrial Electronics, 42(2), 123-130. http://dx.doi.org/10.1109/41.370377

Murakami, T., \& Ohnishi, K. (1993). Torque sensorless control in multidegree-of-freedom manipulator. IEEE Transactions on Industrial Electronics, 40(2), 259-265. http://dx.doi.org/10.1109/41.222648

Ohnishi, K., Katsura, S., \& Shimono, T. (2010). Motion control for real world haptics. IEEE Industrial Electronics Magazine, 4, 16-19. http://dx.doi.org/10.1109/MIE.2010.936761

Prasanga, K., et al. (2012). Achievement of real haptic sensation with tendon driven segregated jaws for laparoscopic forceps. ICIAFS 2012 - Proceedings: 2012 IEEE 6th International Conference on Information and Automation for Sustainability, 65-70. http://dx.doi.org/10.1109/ICIAFS.2012.6419883

Sarlyildiz, E., \& Ohnishi, K. (2014). A comparison study for force sensor and reaction force observer based robust force control systems. 2014 IEEE 23rd International Symposium on Industrial Electronics (ISIE), 1156-1611. http://dx.doi.org/10.1109/ISIE.2014.6864777 
Shimono, T., \& Yamashita, T. (2012). A suppression of cut-off frequency fluctuation based on workspace observer with estimated equivalent mass for mdof bilateral system. The 2012 IEEE/ASME International Conference on Advanced Intelligent Mechatronics, 320-325. http://dx.doi.org/10.1109/AIM.2012.6265972

Tai, H., \& Murakami, T. (2010). Equivalent mass matrix based bilateral control for multi-degrees-of-freedom Systems. International Workshop on Advanced Motion Control, AMC, 343-348. http://dx.doi.org/10.1109/AMC.2010.5464107

Tanida, K., et al. (2012). Function separation for 2-DOF haptic surgical forceps robots driven by multi brive linear motors. The 12th IEEE International Workshop on Advanced Motion Control, AMC, 1-6. http://dx.doi.org/10.1109/AMC.2012.6197134

Togashi, N., et al. (2014). Experimental comparison of design methods for equivalent mass matrix in motion control system based on workspace observer. AMC2014, 669-674. http://dx.doi.org/10.1109/AMC.2014.6823361

Wei, L. J., Shukor, A. Z. H., \& Jamaluddin, M. H. (2016). Investigation on the effects of outer-loop gains , inner-loop gains and variation of parameters on bilateral teleoperation control system using geared DC-motor. International Journal of Mechanical \& Mechatronics Engineering IJMME-IJENS, 16(01), 5469. Retrieved from http://ijens.org/Vol_16_I_01/161601-2929-IJMME-IJENS.pdf

Wei, L. J., Shukor, A. Z. H., \& Jamaluddin, M. H. (2015). Workspace control of two link planar robot using Micro-Box 2000. Jurnal Teknologi, 77(20), 9-18. http://dx.doi.org/10.11113/jt.v77.6545

\section{Copyrights}

Copyright for this article is retained by the author(s), with first publication rights granted to the journal.

This is an open-access article distributed under the terms and conditions of the Creative Commons Attribution license (http://creativecommons.org/licenses/by/3.0/). 